INRA Prod. Anim., 1994, 7 (3), 177 - 189.
C. DEMARQUILLY

INRA Unité de la Valeur Alimentaire Theix 63122 St Genès Champanelle

\section{Facteurs de variation de la valeur nutritive du maïs ensilage}

\author{
Depuis quelques années, les superficies consacrées à la culture du maïs \\ ont connu une progression spectaculaire tant en France que dans les \\ autres pays européens. Dans les régions où sa culture est possible, \\ l'ensilage de maïs est devenu la base de l'alimentation hivernale des \\ bovins ayant des besoins de production élevés (taurillons, vaches \\ laitières). Il est donc important de faire le point sur les facteurs de \\ variation de sa valeur nutritive.
}

\title{
Résumé
}

La composition morphologique de la plante de maïs évolue jusqu'au stade vitreux du grain (35 \% de matière sèche (MS) dans la plante entière), le grain a alors atteint sa maturité physiologique et représente de 40 à $50 \%$ de la MS. La composition chimique évolue relativement peu après la floraison sauf la teneur en amidon qui augmente au détriment de la teneur en glucides solubles. La digestibilité de la matière organique (dMO) et l'ingestibilité, mesurées sur des moutons, d'un maïs donné évoluent peu après le stade laiteux; elles ont été en moyenne respectivement de $71,5 \pm 2,2$ et de $52,5 \pm 7,2 \mathrm{~g} \mathrm{MS} / \mathrm{kg} \mathrm{P} 0,75$ pour les 20 maïs sur pied que nous avons étudiés entre 1964 et 1971 . La dMO a été en moyenne de 72,6 et a varié de 67 à 78 pour les 15 hybrides normaux étudiés par le club digestibilité en 1987 et 1988 . Si on excepte les maïs bm, les variations de digestibilité observées entre variétés cultivées la même année dans un lieu donné sont faibles et du même ordre de grandeur que celles observées pour la même variété entre lieux ou années.

De part sa teneur élevée en MS, sa teneur en glucides solubles non limitante et surtout son pouvoir tampon faible, le maïs se conserve très bien sous forme d'ensilage à condition d'être haché finement. La dMO de l'ensilage est identique à celle de la plante mise en silo. Il en est pratiquement de même de son ingestibilité chez le mouton, du moins quand l'ensilage est correctement complémenté, notamment en azote. En revanche la quantité ingérée par les bovins augmente avec la teneur en MS jusqu'à ce que celle-ci atteigne $35 \%$ environ.

La teneur en MS de l'ensilage de maïs est donc le facteur essentiel de la quantité d'énergie nette ingérée par les bovins. Chez le bovin à l'engrais, il a été bien montré que si le gain de poids vif augmente avec la teneur en MS de l'ensilage de maïs, l'efficacité alimentaire, donc vraisemblablement la valeur énergétique, reste constante. Il semble en être de même chez la vache laitière.

A même teneur en MS, les quantités ingérées et les performances des bovins à l'engrais et des vaches laitières, mais aussi l'efficacité alimentaire, donc la valeur énergétique, varient dans le même sens que la digestibilité de l'ensilage.

Les performances animales ne sont guère affectées par les dispositifs (grille, rouleaux...) qui permettent de diminuer la présence de grains entiers dans les bouses. En revanche, la valorisation énergétique effective (calculée à partir des performances) des rations à base d'ensilage de maïs dépend de la nature et de l'importance de la complémentation énergétique. 
La composition, la digestibilité, l'ingestibilité et, de façon plus globale, la valeur nutritive des fourrages conservés, dépendant d'abord de celles des fourrages sur pied au moment de la récolte, nous allons d'abord traiter de la plante sur pied.

\section{1 / La plante sur pied}

\section{1 / Composition morphologique}

L'évolution de la composition morphologique de la plante de maìs avec le stade de végétation a fait l'objet de nombreux travaux et est maintenant bien connue. Dans des conditions

Tableau 1. Evolution avec le stade de végétation de la composition morphologique et chimique du maïs sur pied (INRA - Laboratoire des Aliments).

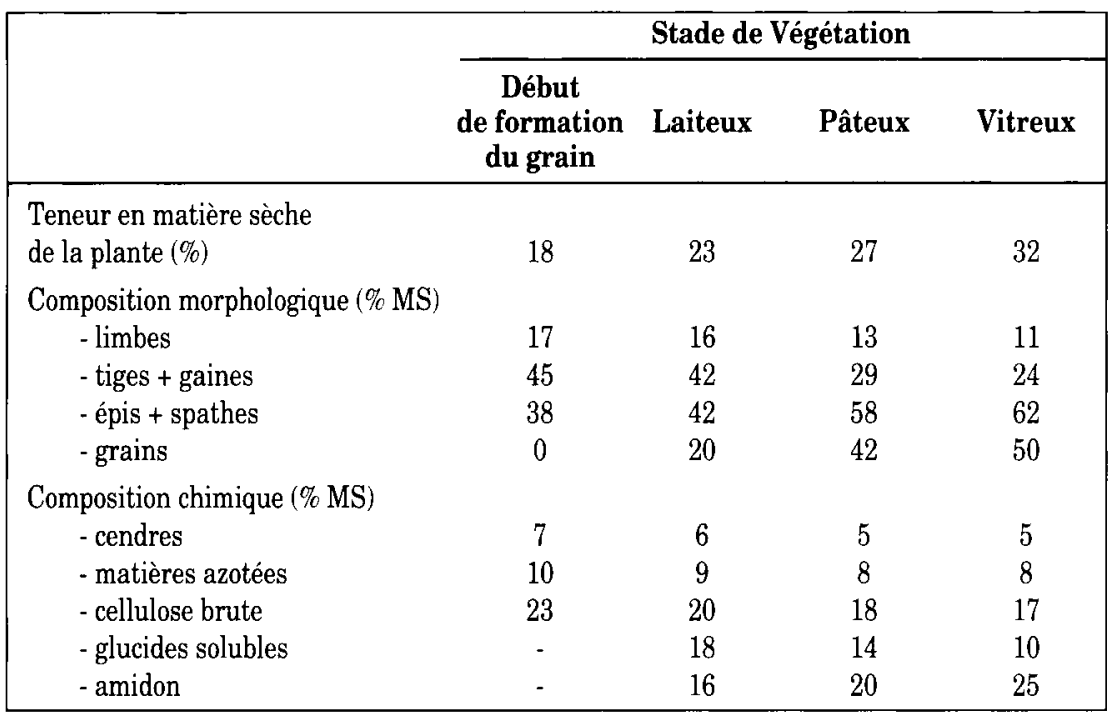

Figure 1. Relation entre les teneurs en glucides solubles et en amidon (méthode Ewers) de la plante de maïs après la floraison (J. Andrieu et al, résultats non publiés).

Teneur en glucides solubles ( $\mathrm{g} / \mathrm{kg} \mathrm{MS}$ )

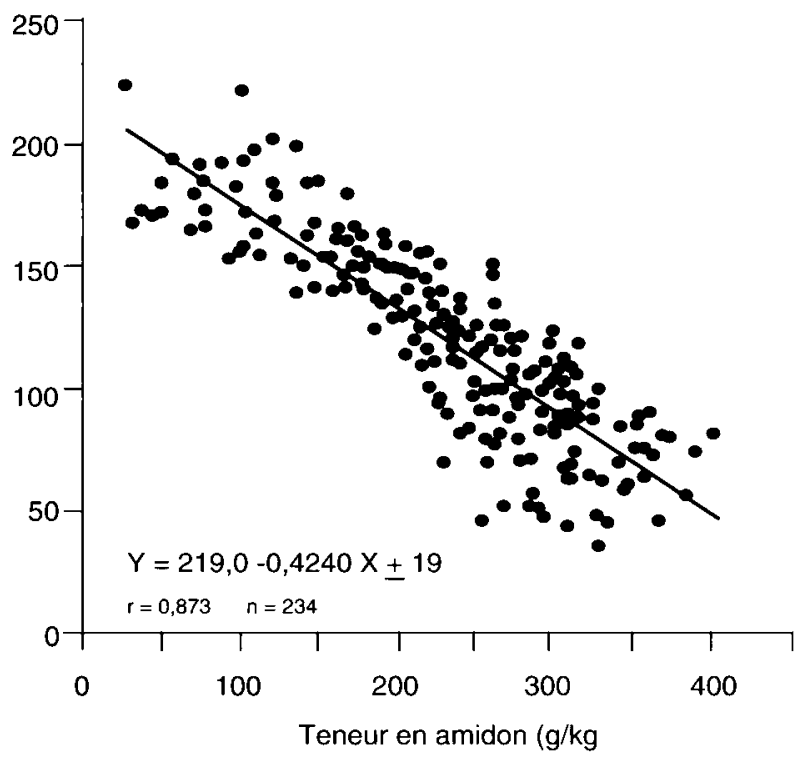

de culture normales, la composition morphologique évolue jusqu'au début du stade vitreux (30-33\% de la matière sèche dans la plante) puis reste pratiquement constante (tableau 1). La part de l'épi (avec ses spathes) passe, suivant les essais, de 20-25\% à la floraison à environ 60-70\% au stade vitreux du grain. Le grain représente alors de 45 à $55 \%$ de la matière sèche de la plante. A l'approche de la maturité, la composition morphologique est relativement peu variable dans le cas des variétés précoces commerciales actuelles. Ses variations ne deviennent importantes que dans des conditions extrêmes, notamment la proportion d'épi et de grain peut rester faible en cas de sécheresse marquée au moment de la floraison.

\section{2 / Composition chimique (tableau 1)}

Entre la floraison et le stade vitreux, la teneur en matière sèche augmente régulièrement : elle passe de 14-16 à 33-35\%. Cette augmentation $(0,35$ point/jour dans les conditions de Clermont-Ferrand) résulte essentiellement de l'accroissement de la part de l'épi dans la plante et de la teneur en matière sèche de l'épi (Andrieu 1969). Dans le même temps, les teneurs en cendres, matières azotées et cellulose brute diminuent légèrement, tandis que la teneur en glucides solubles diminue rapidement au bénéfice de la teneur en amidon. Il existe donc des relations plus ou moins étroites entre certains constituants chimiques ou morphologiques de la plante, notamment entre les teneurs en amidon et en glucides solubles (figure 1), les teneurs en grain et en cellulose brute et surtout les teneurs en grains et en amidon (figure 2) (Andrieu et al 1993). Aux stades où il est normalement ensilé, le maïs est pauvre non seulement en azote, mais aussi en minéraux et oligo-éléments.

\section{3 / Digestibilité et ingestibilité}

Dans nos premiers essais effectués de 1964 à 1971 sur des moutons alimentés à volonté, la digestibilité de la matière organique et l'ingestibilité ont été respectivement de 71,5 $\pm 2,2 \%$ et de $52,5 \pm 7,2 \mathrm{~g}$ de $\mathrm{MS} / \mathrm{kg} \mathrm{P0,75}$ pour les 20 maïs étudiés. Pour un maîs donné, elles n'ont pas varié significativement avec le stade de maturité du grain (figure 3). L'augmentation dans la plante entière de l'épi, dont la digestibilité est élevée et pratiquement constante (de l'ordre de $85 \%$ ) (Demarquilly 1969, Gross 1980, Deinum et al 1984) compense la diminution de la digestibilité du reste de la plante. Celle-ci diminue de 70 à $60 \%$ environ entre les stades laiteux et vitreux du grain. Cette constante de la digestibilité de la plante entière a été retrouvée dans les autres études réalisées in vivo sur ce sujet (Harris 1965, Noller et al 1965, Davis et Bowden 1969). Dans les essais plus récents effectués en 1987 et 1988 en 7 lieux en France, INRA Theix, Rennes, Lusignan et le Pin aux Haras, INRA ENSAA Dijon, ITCF la Jaillère et ENSAT Toulouse dans le cadre du "club Digestibilité" (Andrieu et al 1993) sur des moutons alimentés en quantité limitée, la digestibilité de la matière orga- 
Figure 2. Relation entre la teneur en grain de la plante de maïs et d'une part sa teneur en cellulose brute, d'autre part sa teneur en amidon (d'après Andrieu et al 1993).

Teneur en grain ( $g / \mathrm{kg} \mathrm{MS)}$

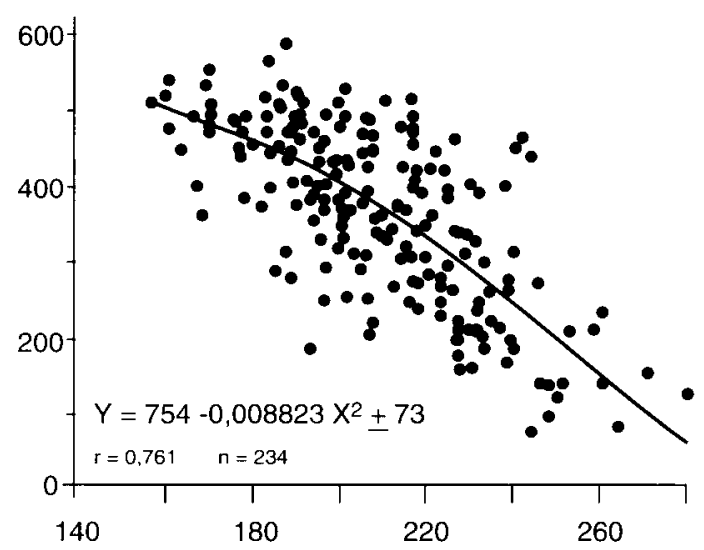

Teneur en cellulose brute ( $\mathrm{g} / \mathrm{kg} \mathrm{MS})$

\section{Teneur en grain ( $g / \mathrm{kg} \mathrm{MS)}$}

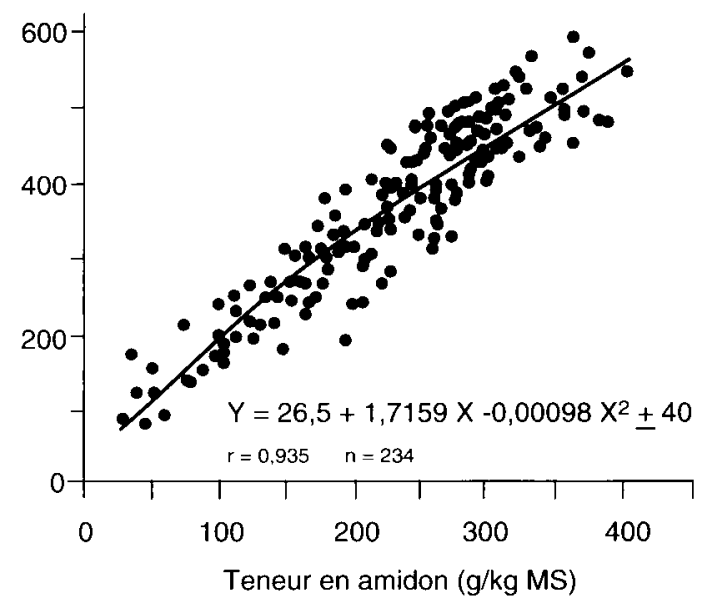

Figure 3. Influence du nombre de jours écoulés depuis la floraison femelle sur la digestibilité du mais sur pied (essais effectués entre 1964 et 1971).

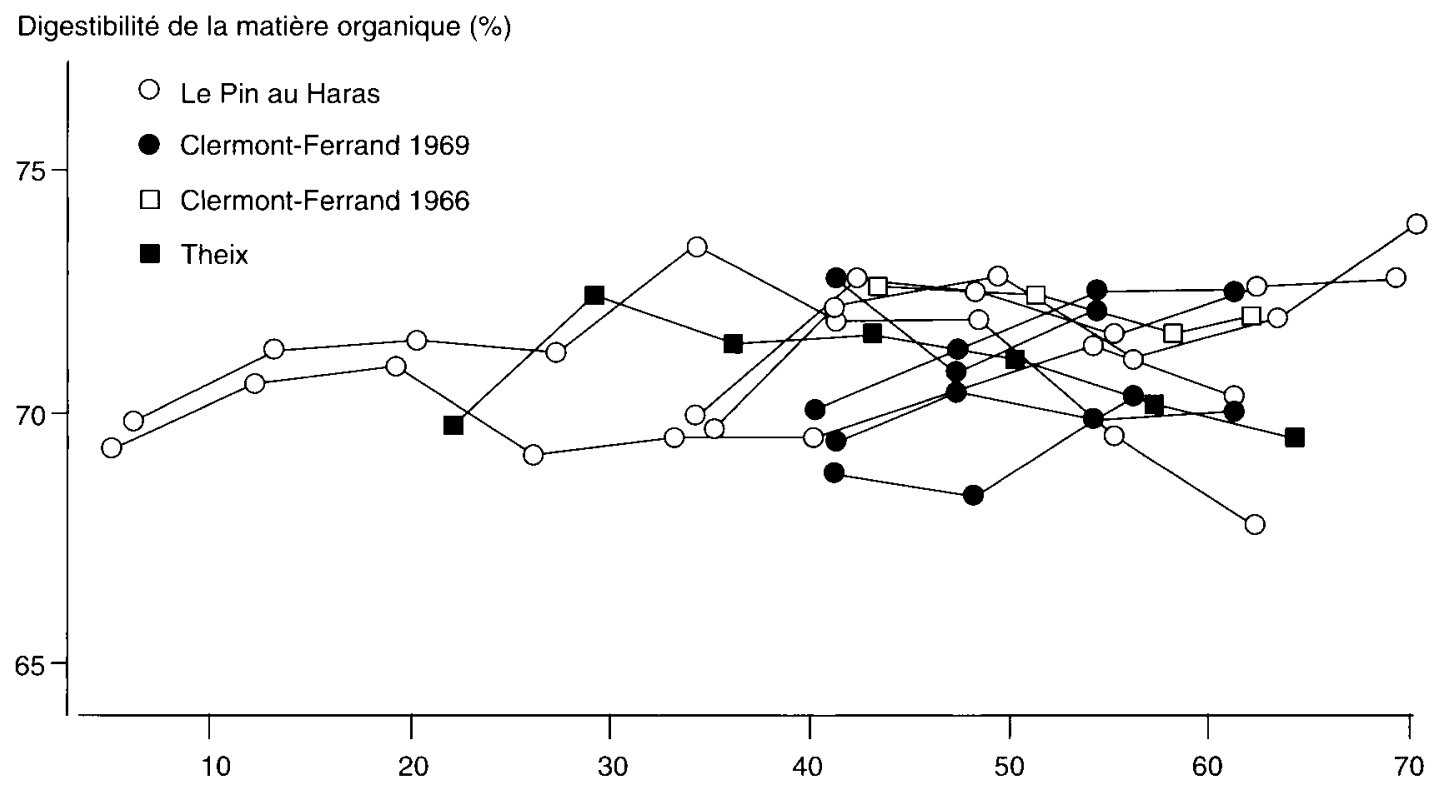

Nombre de jours écoulés depuis la floraison femelle

nique a été en moyenne du même ordre (71,7\%, moyenne de 234 mesures de digestibilité) pour les 15 hybrides normaux (ne portant pas le gène bm3) que dans les premiers essais, mais a évolué de façon différente avec le stade de maturité du grain selon les lieux d'étude. Elle est restée constante dans les trois lieux à climat plus océanique (Le Pin aux Haras, Rennes, La Jaillère) alors qu'elle a augmenté de façon importante et significative jusqu'à environ $35 \%$ de MS et a eu tendance à diminuer ensuite dans les 4 lieux à climat plus continental. (Clermont- Ferrand, Lusignan, Dijon et Toulouse) (figure 4).

Si la digestibilité du mais est de l'ordre de $72 \%$, elle peut varier d'un maïs à l'autre : elle a varié de 69,1 à $76,7 \%$ pour les 20 maïs étu- diés entre 1964 et 1971 et de 67 à $78 \%$ pour les 15 hybrides normaux étudiés en 1987 et 1988. Les variations de digestibilité observées entre hybrides cultivés la même année dans un lieu donné sont faibles, rarement significatives, ne se retrouvent pas forcément une autre année ou dans un autre lieu de culture, et sont du même ordre de grandeur que celles observées pour le même hybride entre lieux ou années (tableau 2). Quand elles existent, les différences de digestibilité ont pu parfois être reliées au pourcentage de grains ou d'épis à un stade donné quand ce pourcentage était très différent entre maïs (Demarquilly 1969), mais résultent le plus souvent de différences dans la digestibilité des parois végétales, donc des parties "non grains" de la plante. Cela est bien montré par l'absence totale de liaison entre la
La dMO du maïs plante entière varie peu, l'accroissement $d \hat{u}$ à l'épi compensant la diminution du reste de la plante. 
Figure 4. Evolution, en fonction du stade, de la digestibilité de la matière organique des mais étudiés en vert en 1987 dans 2 lieux différents (Andrieu et al 1993).

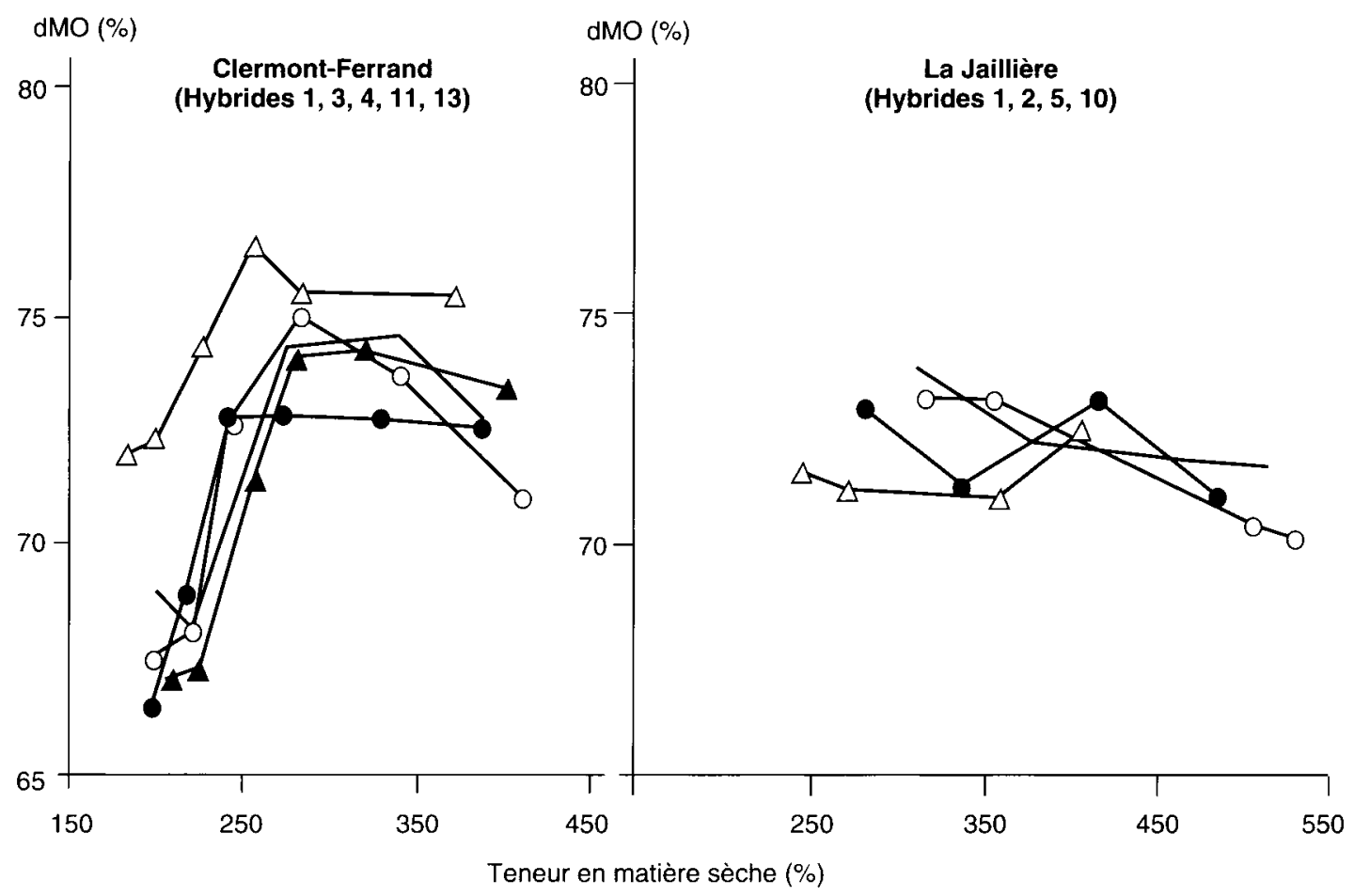

Tableau 2. Digestibilité moyenne de la matière organique (dMO) des hybrides de mais (codés 1, 3, 4, 11 et 13) étudiés à Clermont Ferrand et de l'hybride (codé 1) étudié dans différents lieux en 1987 et 1988 (J. Andrieu et al, résultats non publiés).

\begin{tabular}{|c|c|c|c|c|c|c|}
\hline & \multicolumn{3}{|c|}{1987} & \multicolumn{3}{|c|}{1988} \\
\hline $\begin{array}{l}\mathrm{N}^{\circ} \text { hybrides étudiés } \\
\text { à Clermont-Ferrand }\end{array}$ & $\mathrm{N}^{(1)}$ & \% $\mathrm{MS}^{12+}$ & $\mathrm{dMO} \%$ & $\mathrm{~N}$ & $\% \mathrm{MS}$ & $\mathrm{dMO} \%$ \\
\hline 1 & 4 & 31,4 & $73,3^{\mathrm{a}}$ & 5 & 29,8 & $70,1^{\text {a }}$ \\
\hline 3 & 4 & 31,3 & $73,3^{\mathrm{a}}$ & 4 & 30,2 & $69,3^{\mathrm{a}}$ \\
\hline 4 & 3 & 34,3 & $73,2^{\mathrm{a}}$ & 4 & 31,5 & $72,2^{\mathrm{a}}$ \\
\hline 11 & 3 & 32,8 & $72,7^{\mathrm{a}}$ & 4 & 31,8 & $70,8^{\mathrm{a}}$ \\
\hline 13 & 3 & 30,3 & $75,8^{\mathrm{b}}$ & 3 & 29,9 & $74,9^{b}$ \\
\hline Hybride 1 étudié à & $\mathrm{N}$ & $\% \mathrm{MS}$ & $\mathrm{dMO} \%$ & $\mathrm{~N}$ & $\% \mathrm{MS}$ & $\mathrm{dMO} \%$ \\
\hline Clermont- Ferrand & 4 & 31,4 & $73,3^{\mathrm{a}}$ & 5 & 29,8 & $70,1^{\mathrm{a}}$ \\
\hline Lusignan & 3 & 31,7 & $76,2^{b}$ & 2 & 29,0 & $73,2^{b}$ \\
\hline Le Pin aux Haras & 4 & 30,1 & $72,2^{\mathrm{a}}$ & 5 & 28,8 & $69,9^{\mathrm{a}}$ \\
\hline La Jaillère & 4 & 41,8 & $72,4^{\mathrm{a}}$ & 4 & 34,0 & $70,1^{\text {a }}$ \\
\hline
\end{tabular}

Les écarts de dMO entre hybrides cultivés dans un même lieu sont similaires aux écarts observés pour un même hybride cultivé en différents lieux.

(1) $\mathrm{N}$ = nombre d'échantillons étudiés (1 échantillon = 1 semaine de mesure)

(2) \% MS = teneur en matière sèche moyenne des différents échantillons étudiés.

digestibilité de la matière organique de la plante de maïs et sa teneur en amidon, même quand cette dernière est élevée et correspond donc à des maïs dont la composition morphologique évolue peu (figure 5), et surtout par la liaison très étroite observée entre la digestibilité de la matière organique et la teneur en parois non digestibles (NDFnd) pour 99 des échantillons de maïs plante entière étudiés en 1987 et 1988 (figure 6). La variation de 1 à plus de $2(120$ à $260 \mathrm{~g} / \mathrm{kg} \mathrm{MS})$ de la teneur en NDFnd résulte en partie de celle de la teneur en NDF (donc du pourcentage de grain dans la plante) mais surtout de la digestibilité du NDF provenant essentiellement des parois des feuilles et des tiges. La digestibilité de la matière organique de l'hybride $\mathrm{bm} 3$, plus élevée $(+5$ points) que celle des hybrides normaux, s'explique d'ailleurs par la digestibilité plus élevée de son NDF (en moyenne 69,0 $\pm 4,1$ contre $56,6 \pm 7,1$ ) alors que sa teneur en NDF est non significativement différente (450 contre $470 \mathrm{~g} / \mathrm{kg} \mathrm{MS}$ ).

Les derniers essais ont par ailleurs permis : - à Andrieu et al (1993) d'améliorer la prévision de la teneur en énergie brute du maïs en vert ( $\mathrm{EB}$ en $\mathrm{kcal} / \mathrm{kg}$ de matière organique) à partir de la teneur en matières azotées totales 
Figure 5. Relation entre la digestibilité de la matière organique des maïs plante entière en vert et leurs teneurs en amidon (J. Andrieu et al, résultats non publiés).

Digestibilité de la matière organique (dMO, \%)

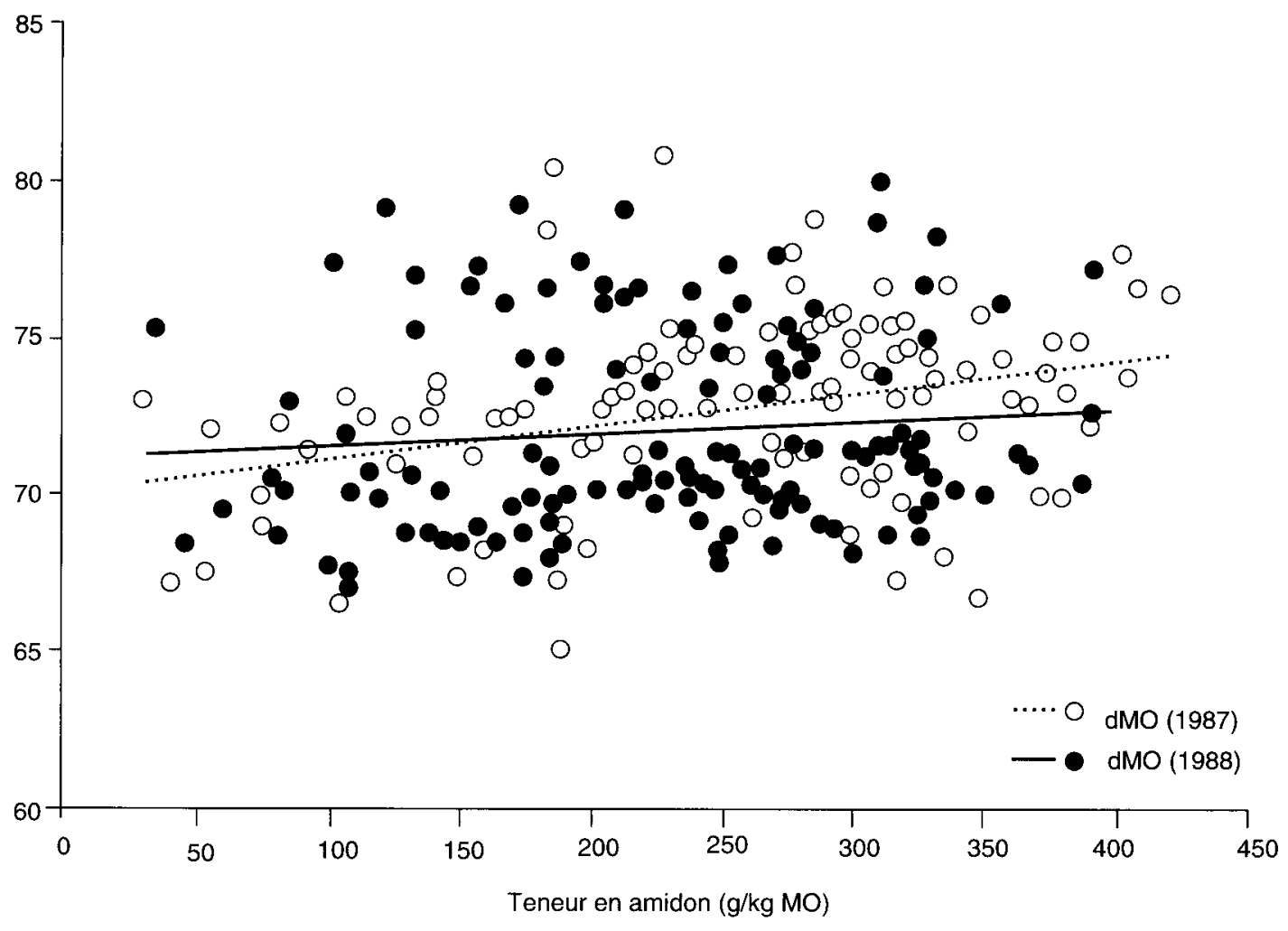

Figure 6. Liaison entre la digestibilité de la matière organique des maïs plante entière en vert et leurs teneurs en parois non digestibles (NDFnd) (Andrieu et al 1993).

Digestibilité de la matière organique (dMO, \%)

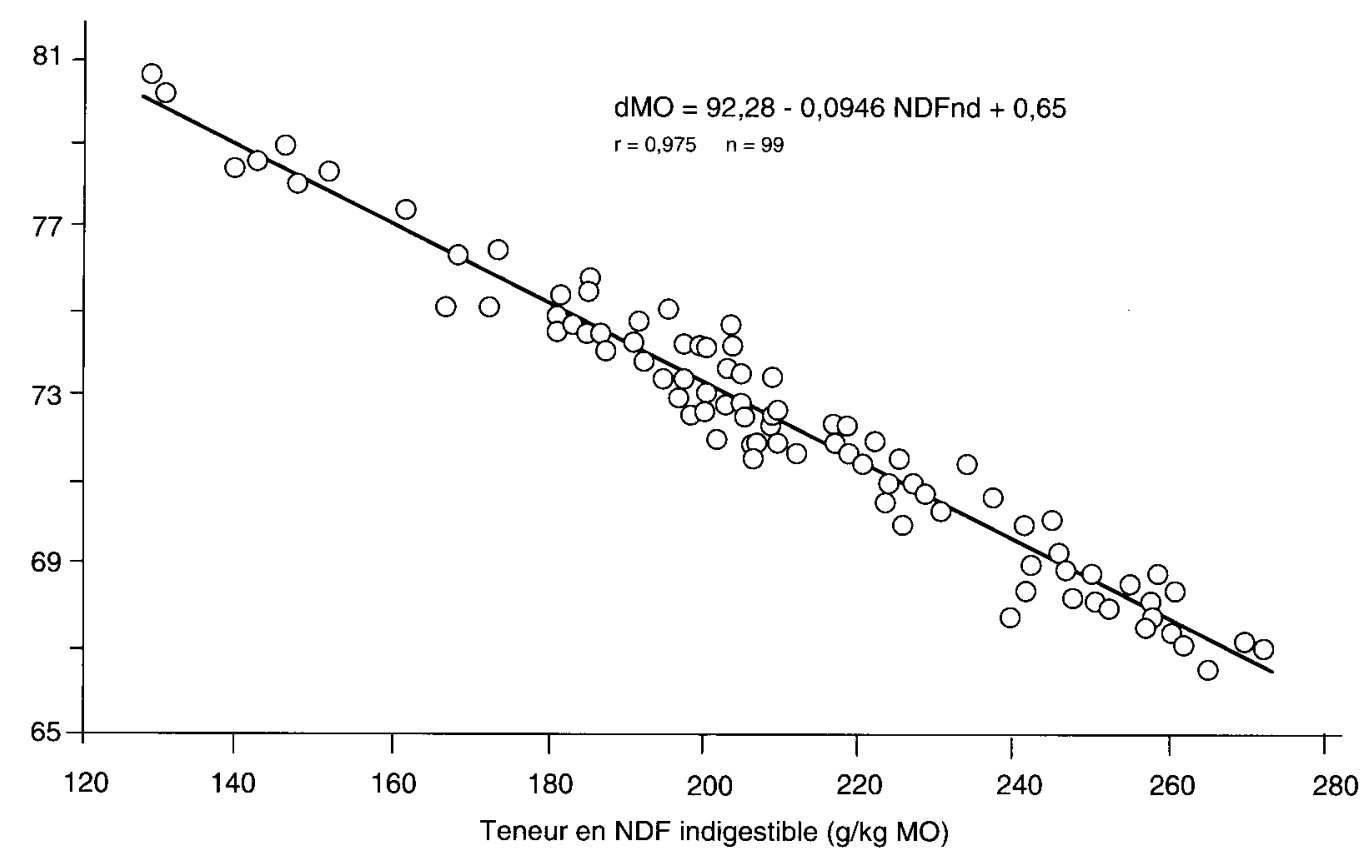

(MAT en $\mathrm{g} / \mathrm{kg}$ matière organique)

$\mathrm{EB}=4487+2,019 \mathrm{MAT} \pm 25$

$\mathrm{r}=0,578$ pour $\mathrm{n}=59$

et de relier la digestibilité de la matière organique (dMO en \%) à la digestibilité de l'énergie (dE en \%)
$\mathrm{dE}=0,9965 \mathrm{dMO}-2,35 \pm 0,22$ $\mathrm{r}=0,998$ pour $\mathrm{n}=37$

- et à Dardenne et al (1993) de proposer toute une série d'équations de prévision de la dMO à partir de critères chimiques ou enzymatiques. La précision n'est satisfaisante qu'en associant différents 
Figure 7. Influence du nombre de jours écoulés depuis la floraison femelle sur la quantité de matière sèche de maïs sur pied ingérée par le mouton (essais effectués entre 1964 et 1971).

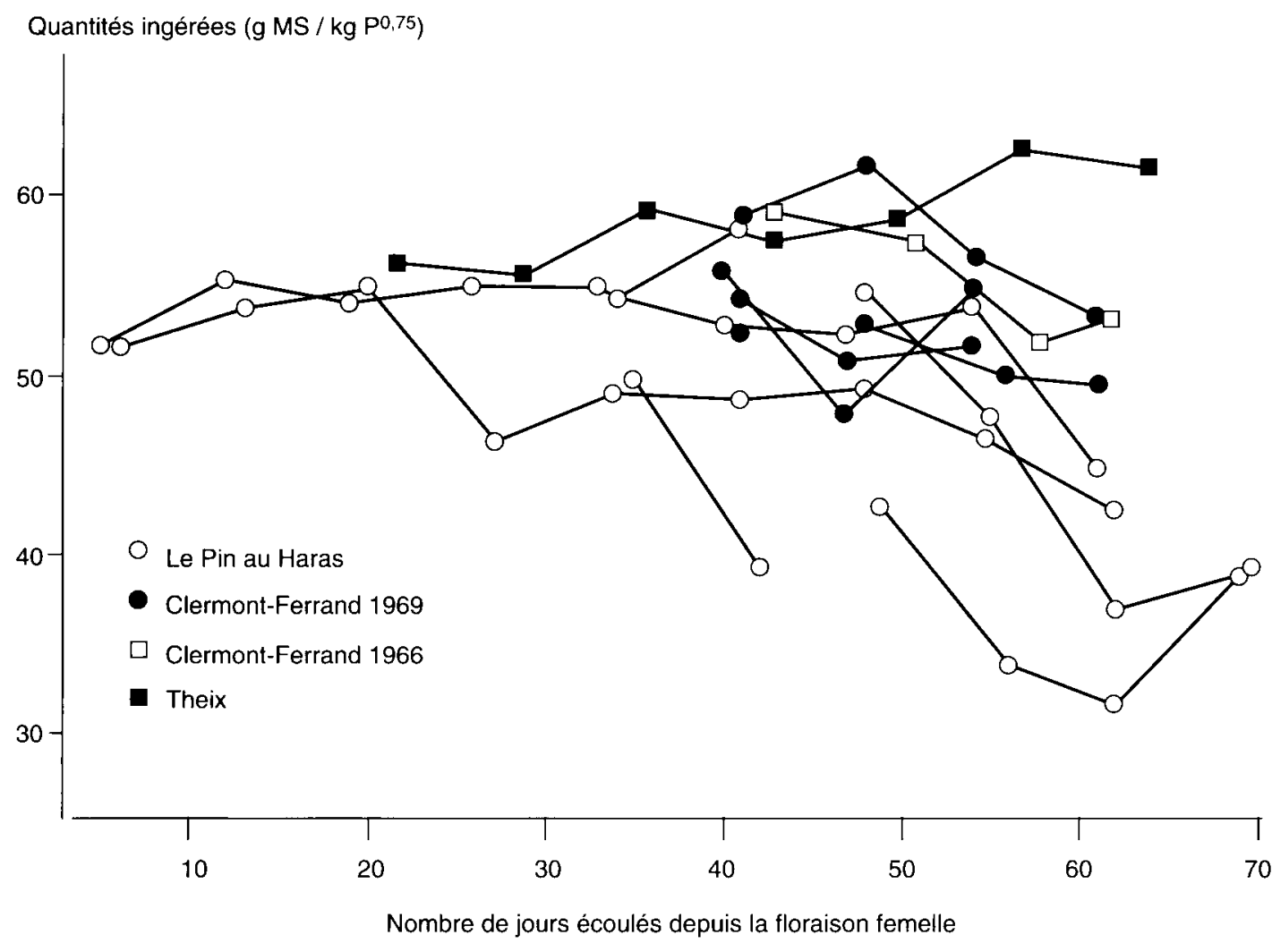

critères, par exemple les teneurs en matières azotées (MAT), en acid detergent fiber $(\mathrm{ADF})$ et en acid detergent lignin (ADL) en $\mathrm{g} / \mathrm{kg} \mathrm{MS}$.

$\mathrm{dMO}=77,7+0,0908 \mathrm{MAT}-0,0188 \mathrm{ADF}-$ $0,3301 \mathrm{ADL} \pm 1,91$

$\mathrm{r}=0,74$ pour $\mathrm{n}=199$

Seule la spectrophotométrie dans le proche infra-rouge permet une amélioration importante de la prévision ( $\pm 1,65$ point).

Lors des premiers essais dans lesquels les maiis ont été distribués à volonté aux moutons, l'ingestibilité (quantité de matière sèche volontairement ingérée) a été nettement plus variable d'un maìs à l'autre que la digestibilité (figure 7). Les différences d'ingestibilité observées entre variétés, lieux ou années n'ont pas pu être reliées à des différences de digestibilité, de composition morphologique (proportion d'épis à l'approche de la maturité) ou de composition chimique (teneurs en matières azotées ou en cellulose brute). Leurs causes sont donc inconnues, mais résultent vraisemblablement, comme pour les fourrages classiques de graminées et de légumineuses (Demarquilly et Chenost 1969), de différences dans les cinétiques de digestion dans le rumen.

\section{4 / Stade optimum de récolte}

La digestibilité et la quantité ingérée étant pratiquement indépendantes du stade, le stade optimum de récolte est celui où la quantité de matière sèche récoltée à l'hectare est maximum, c'est-à-dire au stade vitreux du grain (figure 8). La teneur en MS de la plante (33 -
$35 \%$ ) est alors idéale pour obtenir une bonne qualité de conservation (absence de jus, fermentations réduites) et correspond à une ingestibilité maximum de l'ensilage chez les bovins.

\section{2 / La plante ensilée}

\section{1 / Composition et qualité de conservation}

Le maïs plante entière est certainement un des fourrages qui se conserve le mieux sous forme d'ensilage, si bien que les caractéristiques fermentaires des ensilages, sans conservateur mais correctement préparés, (hachage fin, remplissage rapide du silo...) sont en général excellentes (tableau 3 ). Comme l'ont signalé de nombreux auteurs (cf revue de Coppock et Stone 1968), les fermentations, dans lesquelles la fermentation lactique prédomine largement, donnent naissance, à partir des glucides solubles qui disparaissent en presque totalité, à de l'acide lactique (de 35 à $75 \mathrm{~g} / \mathrm{kg}$ de MS), à un peu d'acide acétique (de 10 à $25 \mathrm{~g} / \mathrm{kg} \mathrm{MS}$ ) et à des alcools (de 50 à $5 \mathrm{~g} / \mathrm{kg} \mathrm{MS}$ ). Elles entraînent un abaissement important $(\leq 4,0)$ et rapide (au bout de 2 à 3 jours seulement) du $\mathrm{pH}$ et, par là, l'inhibition de la fermentation butyrique.

Grâce à cette diminution rapide $\mathrm{du} p H$, les enzymes de la plante ne dégradent qu'une proportion faible des protéines ; dans les 35 comparaisons réalisées dans notre laboratoire, la 
proportion d'azote sous forme soluble est passée en moyenne de $26 \%$ à la mise en silo à seulement $47 \%$ dans l'ensilage. De même, la proportion d'azote sous forme ammoniacale n'est que de l'ordre de 5 à $7 \%$. Cette aptitude remarquable à l'ensilage de la plante de maïs résulte de sa teneur en matière sèche élevée $(\geq 25 \%)$, de sa teneur en sucres suffisante ( $\geq 8-10 \%$, même à des stades avancés de maturité) et surtout à son pouvoir tampon faible (Andrieu et Demarquilly 1974a, Wilkinson et Phipps 1979, Demarquilly 1982).

Comme l'ont observé de nombreux auteurs, l'intensité des fermentations et de la protéolyse diminue quand la teneur en MS augmente, donc que le stade de récolte est plus tardif (tableau 3).

Si l'on excepte la teneur en glucides solubles, la composition chimique classique est peu modifiée : il y a seulement une légère augmentation passive des teneurs en matières azotées, en cellulose brute et en amidon.

\section{2 / Digestibilité et ingestibilité}

Quand la plante de maïs est ensilée à plus de $25 \%$ de MS et que la teneur en MS de l'ensilage est corrigée pour les pertes de produits volatils lors du séchage à l'étuve, la digestibilité de la matière organique de l'ensilage est identique à celle de la plante sur pied au moment de la récolte (Harris 1965, Andrieu et Demarquilly 1974). Pour 4 maïs ensilés à $20 \%$ de MS, J. Andrieu (résultats non publiés) a cependant observé que l'ensilage entraînait une diminution de digestibilité, en moyenne de 3 points, vraisemblablement par suite de la perte dans les jus de constituants très digestibles. Dans ces conditions, il n'est donc pas étonnant que la digestibilité des 72 ensilages de maïs (complémentés en azote car la teneur en matières azotées de l'ensilage est un facteur limitant de la digestibilité si elle est inférieure à $8,5 \%$ : cf Andrieu et Demarquilly 1974) a été en moyenne du même ordre de grandeur :
Figure 8. Evolution du poids sec d'une plante de maïs (moyenne d'un échantillon de 10 plantes) en fonction de l'âge (jours écoulés depuis la floraison femelle) en Limagne (d'après Andrieu 1969).

Poids sec (g)

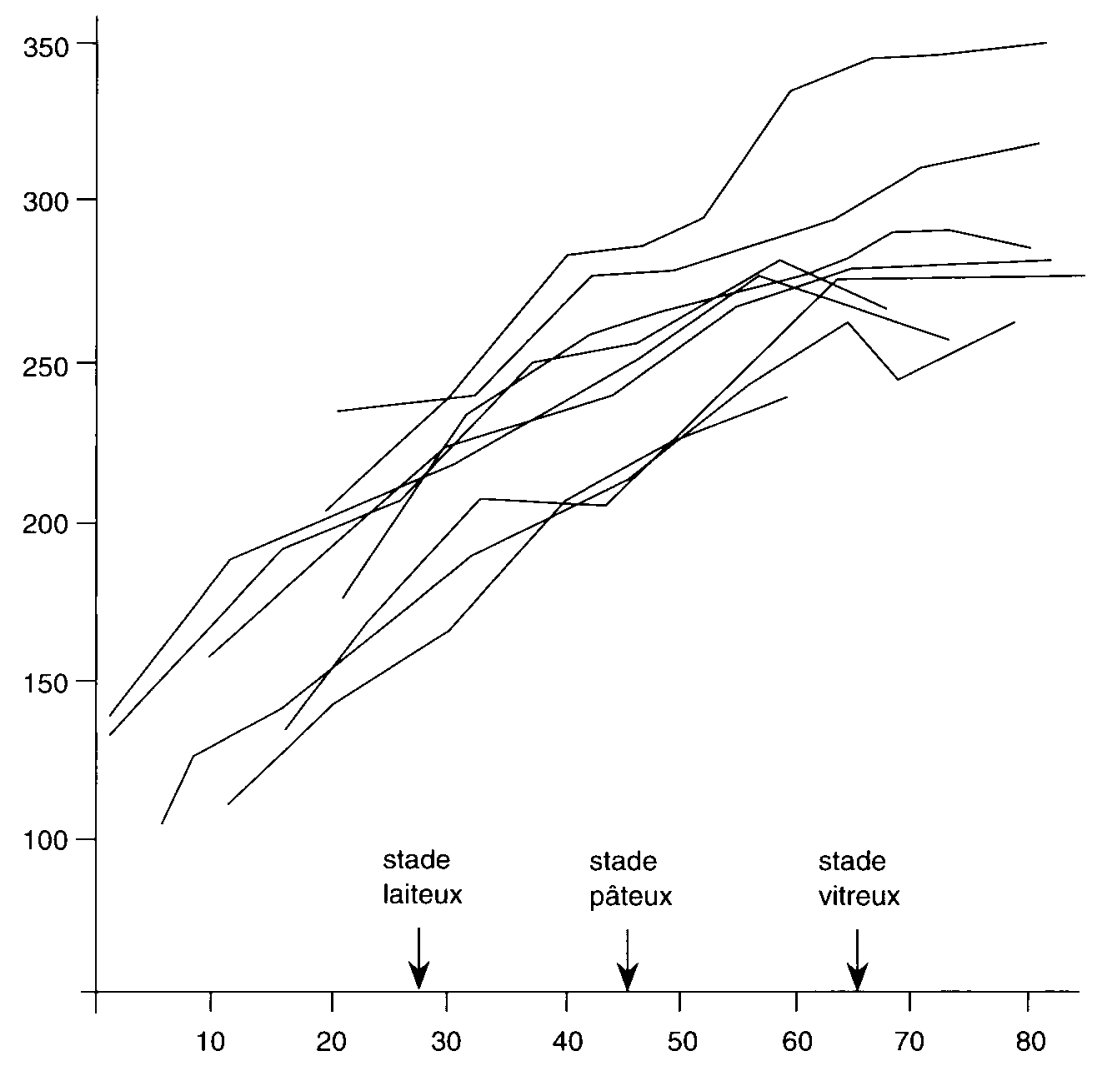

Nombre de jours après la floraison

$70,4 \%$, et a varié dans les mêmes limites (de 65,8 à $76,0 \%$ ) que celle des maïs étudiés en vert. La digestibilité des ensilages préparés à partir d'un maïs donné a été indépendante du stade de récolte comme l'ont montré aussi d'autres auteurs (Coppock et Stone 1968, Johnson et Mc Clure 1968, Hillman 1969 ...).

Tableau 3. Qualité de conservation et valeur alimentaire des ensilages de maïs plante entière avec (+) ou sans (-) addition d'urée et de minéraux à la mise en silo (INRA Laboratoire des aliments).

\begin{tabular}{|c|c|c|c|c|c|c|c|c|c|c|c|}
\hline Teneur en MS (\%) & \multicolumn{2}{|c|}{$20-25$} & \multicolumn{2}{|c|}{ 25-30 } & \multicolumn{2}{|c|}{$30-35$} & \multicolumn{2}{|c|}{$35-40$} & $40-45$ & \multicolumn{2}{|c|}{ Moyenne } \\
\hline Addition urée-minéraux & - & + & - & + & - & + & - & + & + & - & + \\
\hline Nombre & 8 & 14 & 17 & 14 & 25 & 21 & 5 & 14 & 9 & 55 & 72 \\
\hline $\mathrm{pH}$ & 3,75 & 4,05 & 3,75 & 4,05 & 3,80 & 4,05 & 3,95 & 4,09 & 4,25 & 3,79 & 4,08 \\
\hline Azote soluble ( $\%$ de $\mathrm{N}$ total) & 60 & 79 & 51 & 71 & 46 & 60 & 39 & 51 & 51 & 49 & 63 \\
\hline Ammoniac ( $\%$ de $\mathrm{N}$ total) & 6,6 & 8,3 & 5,3 & 14,2 & 5,5 & 12,0 & 5,1 & 12,0 & 11,0 & 5,6 & 11,6 \\
\hline Acide lactique (g/kg MS) & 62,8 & 62,4 & 48,4 & 57,5 & 48,5 & 56,4 & 46,7 & 56,2 & 50,0 & 50,4 & 56,9 \\
\hline Acide acétique (g/kg MS) & 18,8 & 22,0 & 15,5 & 15,4 & 13,4 & 14,1 & 9,3 & 12,7 & 11,1 & 14,5 & 15,2 \\
\hline Acide butyrique ( $\mathrm{g} / \mathrm{kg} \mathrm{MS}$ ) & 0,2 & 0,4 & 0,3 & 1,0 & 0,2 & 0,4 & 0,2 & 0,4 & 0,2 & 0,2 & 0,5 \\
\hline Alcool (g/kg MS) & 48,6 & 48,8 & 27,6 & 31,9 & 12,8 & 8,7 & 4,7 & 7,7 & 3,7 & 21,8 & 20,2 \\
\hline Digestibilité de la $\mathrm{MO}(\%)$ & 68,4 & 68,4 & 69,9 & 70,4 & 70,3 & 71,6 & 69,0 & 70,7 & 70,2 & 69,8 & 70,4 \\
\hline MS ingérée ${ }^{(2)}\left(\mathrm{g} / \mathrm{kg} \mathrm{P}^{0.75}\right)$ & 50,4 & 53,5 & 51,6 & 54,3 & 50,9 & 54,7 & 49,6 & 51,0 & 50,0 & 50,9 & 53,0 \\
\hline
\end{tabular}

"I Un mélange solide, contenant pour moitié de l'urée (46\% d'azote) et pour moitié des minéraux, a été incorporé à la dose de $10 \mathrm{~kg}$ par tonne de maïs frais.

${ }^{\text {[2) }}$ par des moutons

L'ensilage ne modifie pas la dMO du maïs plante entière si sa teneur en MS dépasse $25 \%$. 
Figure 9. Liaison entre la digestibilité de la matière organique des ensilages de maïs récoltés à plus de $28 \%$ de MS et leurs teneurs en grains à la mise en silo (d'après Andrieu 1984).

Digestibilité de la matière organique (dMO, \%)

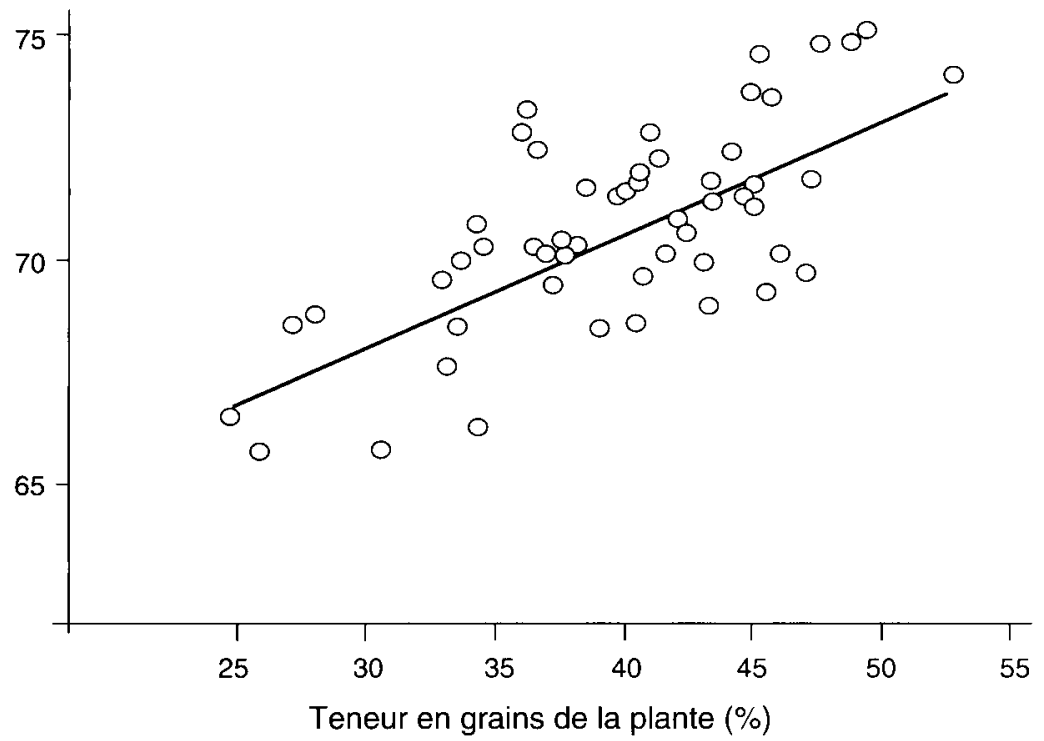

Pour les maïs récoltés plus de 50 jours après la floraison elle a été en moyenne plus élevée $(74 \%)$ pour les maiis riches en épis (plus de $65 \%$ ) et plus faible $(68 \%)$ pour les maïs pauvres en épis parce qu'ayant subi une sécheresse estivale. Pour ces mais il existe donc des relations significatives entre la digestibilité et la teneur en grains (figure 9) et, par là, les teneurs en cellulose brute, ou en amidon (Andrieu 1984). Quoique hautement significatives, ces relations n'expliquent cependant que $50 \%$ des variations de digestibilité observées chez ces maïs. Les variations de la digestibilité des tiges doivent vraisemblablement expliquer les autres $50 \%$. On peut d'ailleurs s'étonner que la prévision de la dMO des ensilages de maïs à partir de la teneur en cellulose brute soit plus précise $(r=0,706)$ que pour les maïs en vert $(r=0,620)$. La raison en est peut être que les ensilages ont été préparés à partir d'un nombre plus restreint de variétés (7) toutes cultivées dans la région de Clermont-Ferrand. De même Rivière (1990), sur un nombre limité ( $\mathrm{n}=14$ ) de maïs en vert d'une teneur en MS supérieure à $27 \%$ correspondant essentiellement à une même variété cultivée de 1982 à 1987 dans un même lieu (La Jaillère), a observé une bonne prévision de la dMO à partir soit de la teneur en amidon (ETR $=0,93$ point) soit de la cellulose brute (ETR = 1,2 point). Il apparaît donc plus facile de prédire la digestibilité des maïs cultivés dans un même lieu (surtout s'il s'agit d'une même variété) que celle des maïs cultivés dans des lieux différents (surtout s'ils correspondent à différentes variétés).

Dans nos essais, l'ingestibilité des maìs chez le mouton n'a pratiquement pas été modifiée par l'ensilage, du moins quand l'ensilage est correctement complémenté, notamment en azote. La quantité de matière sèche d'ensilage de maîs ingérée par le mouton est donc indépendante du stade de récolte et, par là, de la teneur en matière sèche de l'ensilage. En revanche de nombreux auteurs (cf revues de Malterre 1976, Coppock et Stone 1986) ont constaté que l'ingestibilité chez des bovins des ensilages de mais augmentait avec leur teneur en matière sèche, et cela, jusqu'à une teneur de $35 \%$ environ. Cela pourrait être dû au fait que chez les bovins la quantité ingérée augmente quand la teneur en parois végétales diminue au profit de la teneur en grain (puisque teneur en matière sèche et teneur en grain évoluent souvent parallèlement) comme le laissent supposer les résultats de Rémond et Journet (1977) et de Fisher et Fairey (1982) qui observent qu'à teneur en matière sèche très comparable, l'ensilage riche en grain est ingéré par des vaches laitières en plus grande quantité que l'ensilage moins riche en grain. Cependant, et surtout, elle pourrait résulter d'une diminution de l'intensité des fermentations quand la teneur en matière sèche s'accroît, intensité des fermentations à laquelle le bovin est beaucoup plus sensible que le mouton (Dulphy et Michalet-Doreau 1981).

\section{3 / Valeur nutritive et performances des bovins}

La teneur en matière sèche de l'ensilage de maïs est le facteur essentiel de la quantité d'énergie nette ingérée par les bovins et, par là, des performances permises par la ration de base d'ensilage de maïs. Pour des ensilages correspondant à des maïs ayant eu des conditions de végétation normales, l'utilisation du logiciel INRAtion montre qu'une vache de $600 \mathrm{~kg}$ en pleine lactation, produisant $25 \mathrm{~kg}$ de lait $4 \%$ par jour et recevant de l'ensilage de maîs à volonté et un concentré à base d'orge et de tourteau de soja pour couvrir ses besoins énergétiques et azotés, consomme respectivement $12,013,5$ et $15,3 \mathrm{~kg}$ de matière sèche d'ensilage de maïs suivant qu'il est à 25,30 , ou $35 \%$ de MS, l'ensilage couvrant alors les besoins énergétiques, en plus de l'entretien, d'une production de 13,$2 ; 16,2$ et $19,9 \mathrm{~kg}$ de lait $4 \%$. La valeur énergétique de l'ensilage (0,90 UFL $/ \mathrm{kg}$ MS en moyenne) n'est pas modifiée entre 25 et $35 \%$ de MS ce qui ressort aussi des travaux américains sur la production laitière (Huber et al 1965, Owen 1967). De même, les résultats de 10 essais (essentiellement français) effectués sur taurillons et récapitulés par Raymond et al (1986) (tableau 4) montrent qu'entre 25 et $35 \%$ de matière sèche, à une augmentation de teneur en matière sèche de 5 points correspond en moyenne une augmentation de la teneur en grain de $5,7 \%$, de la vitesse de croissance de $4 \%(+41 \mathrm{~g} / \mathrm{jour})$, mais cette dernière résulte essentiellement d'une augmentation $(+4 \%)$ de la quantité d'ensilage ingérée, de sorte que l'indice de consommation (kg MS ingérée/kg de gain de poids vif) et, par là, vraisemblablement la valeur énergétique n'est pas modifiée. L'augmentation du gain de poids vif $(+56 \mathrm{~g} / \mathrm{jour}$, soit $5,3 \%)$ étant cependant plus grande quand on passe de 25 à $30 \%$ de MS que lorsqu'on passe de 30 à $35 \%$ $(+14 \mathrm{~g} /$ jour, soit $1,3 \%)$, les auteurs considèrent qu'il y a peu d'intérêt à dépasser $30 \%$ de MS pour l'engraissement des taurillons. 
Tableau 4. Influence d'une augmentation de la teneur en matière sèche de l'ensilage de maïs de 5 points sur la teneur en grains, la quantité ingérée et les performances des taurillons (d'après Raymond et al 1986).

\begin{tabular}{|lccccc|}
\hline Teneur en MS du témoin & $\begin{array}{c}\text { \% grain de } \\
\text { l'ensilage }\end{array}$ & $\begin{array}{c}\text { Quantité } \\
\text { ingérée } \\
(\%)\end{array}$ & $\begin{array}{c}\text { Gain de poids vif } \\
(\mathbf{g})\end{array}$ & $\begin{array}{c}\text { kg MS par } \\
\text { kg gain } \\
(\%)\end{array}$ \\
\hline $\begin{array}{l}<30 \% \\
(7 \text { essais })\end{array}$ & $+6,6$ & $+4,9$ & +56 & $+5,3$ & 0,6 \\
\hline $\begin{array}{l}>30 \% \\
(3 \text { essais })\end{array}$ & $+3,6$ & $+2,8$ & +14 & $+1,3$ & 1,7 \\
\hline $\begin{array}{l}\text { Total (pour } 5 \text { points) } \\
(10 \text { essais) }\end{array}$ & $+5,7$ & 4,0 & +41 & $+4 \%$ & 0,9 \\
\hline
\end{tabular}

A même teneur en matière sèche, les performances des vaches laitières et des bovins à l'engrais, mais aussi l'efficacité alimentaire, donc la valeur énergétique de l'ensilage, varient dans le même sens que la digestibilité de l'ensilage. Cela a été montré, tant chez les vaches laitières (Rémond et Journet 1977) que chez les taurillons ou les bouvillons (cf synthèse de Raymond et al 1986 ; tableau 5), dans les comparaisons entre des maïs récoltés au même stade, mais très différents par leur teneur en grains (donc de digestibilité différente, bien que celle-ci n'ait pas toujours été mesurée) soit par suite d'accidents climatiques liés à la sécheresse estivale, soit par suite de densité de semis très différente. Cela a été montré aussi dans les comparaisons entre des ensilages de maîs normaux et des mêmes maïs, de même teneur en matière sèche et de même richesse en grains, mais plus digestibles parce que porteurs du gène bm3. Dans les 6 essais effectués à l'INRA de Lusignan (Barrière et al 1985), des taurillons recevant les ensilages de maïs bm3 ont, à même niveau de complémentation, ingéré $7,7 \%$ de matière sèche d'ensilage en plus, ont accru de $198 \mathrm{~g} /$ jour $(+15,7 \%)$ leur gain de poids vif et de $92 \mathrm{~g}(+11,5 \%)$ leur gain de carcasse. L'efficacité alimentaire, exprimée en gain de carcasse par $\mathrm{kg}$ de MS ingérée, a été accrue de $4,2 \%$, valeur très comparable à celle de l'augmentation de la valeur énergétique nette $(+0,03 \mathrm{UFV} / \mathrm{kg} \mathrm{MS}$, soit $+4 \%)$ estimée à partir des mesures de digestibilité sur moutons (+ 1,6 points) (tableau 6 ). En revanche on ignore quelles seraient les quantités ingérées et les performances des bovins qui recevraient des ensilages de mails récoltés au même stade et dont les teneurs en grains seraient différentes, mais qui seraient de même digestibilité parce que la digestibilité des parties "non grains" serait différente.

Si la finesse de hachage est un facteur important de la qualité de conservation et de l'ingestibilité des ensilages, il n'est pas utile qu'elle soit excessive bien que certains grains entiers se retrouvent dans les fêces. Les différents essais ayant comparé des ensilages de maïs simplement hachés aux mêmes ensilages dont la proportion de grains intacts a été considérablement diminuée par l'emploi de rouleaux ou de grille, montrent que l'éclatement des grains n'améliore pas les performances des
Tableau 5. Influence d'une augmentation de 5 points de la teneur en grain de l'ensilage de maiss sur les quantités ingérées et les performances des taurillons et bouvillons (d'après Raymond et al 1986).

\begin{tabular}{|lcc|}
\hline & $\begin{array}{c}\text { Taurillons } \\
\text { 3 essais }\end{array}$ & Bouvillons \\
& 12 essais \\
\hline Quantité ingérée (\%) & $-2,8$ & $-0,2$ \\
Gain de poids vif & & \\
- en g & +12 & +62 \\
- en \% & +1 & $+6,3$ \\
kg MS / kg gain de poids vif $(\%)$ & $-3,0$ & $-4,7$ \\
kg MS / kg gain de carcasse (\%) & $-4,0$ & \\
\hline
\end{tabular}

animaux à l'engrais quand le hachage est normal et très peu quand le hachage est insuffisant comme le montrent les résultats de l'ITCF présentés au tableau 7 , les croissances et les quantités ingérées étant non significativement différentes. Les résultats de De Brabender et al (1990) (tableau 8) montrent l'intérêt d'un hachage normal du maïs ( $5 \mathrm{~mm}$ ) par comparaison à un hachage insuffisant $(16 \mathrm{~mm})$ sur les quantités d'ensilage ingérées par les vaches laitières. Cet intérêt ressort aussi des essais de l'EDE de Vendée rapportés par Cabon (1987) qui préconise, pour les vaches laitières, la granulométrie suivante des ensilages de maïs :

$\begin{array}{lr}\text { particules } \geq 20 \mathrm{~mm} & 1 \% \\ \text { particules entre } 10 \text { et } 20 \mathrm{~mm} & 9 \% \\ \text { particules entre } 6 \text { et } 10 \mathrm{~mm} & 45 \% \\ \text { particules }<6 \mathrm{~mm} & 45 \%\end{array}$

Cette granulométrie peut être obtenue avec des ensileuses sans grille ni rouleau éclateur de grains avec un réglage théorique de hachage de $5 \mathrm{~mm}$ et des couteaux bien affutés et pour celles munies de grille ou d'éclateur avec un réglage de hachage de 7 à $9 \mathrm{~mm}$. En revanche, les essais américains montrent que les hachages trop fins, obtenus surtout par des rehachages ou des broyages lors de la mise en silos, entraînent dans certains cas des baisses de taux butyreux du lait (Huber et al 1966, Gordon et al 1968, Miller et al 1969).

Pour une même teneur en matière sèche et une même finesse de hachage, des essais récents entrepris au domaine INRA d'Orcival montrent que les quantités de matière sèche
Chez les bovins, les quantités ingérées augmentent jusqu'à une teneur en MS de l'ensilage de $35 \%$. 
Tableau 6. Influence du gène bm3 sur la digestibilité (moutons) de l'ensilage de maïs et sur la quantité ingérée et les performances des taurillons à même niveau de complémentation (1 $\mathrm{kg}$ MS tourteau de soja) (d'après Barrière et al 1985).

\begin{tabular}{|lccc|}
\hline Ensilage de maïs & normal & bm3 & Ecart (\%) \\
\hline Teneur en MS & 36,2 & 35,1 & \\
Teneur en grain & 44,1 & 42,2 & \\
Digestibilité de la matière organique & 71,6 & 73,2 & $1,6(2,2)$ \\
Matière sèche ingérée $(\mathrm{kg} / \mathrm{j}$ par animal) & 7,38 & 7,95 & $0,57(7,7)$ \\
Gain de poids vif $(\mathrm{g} / \mathrm{j})$ & 1265 & 1463 & $198(15,7)$ \\
Gain de carcasse $(\mathrm{g} / \mathrm{j})$ & 798 & 890 & $92(11,5)$ \\
Gain de poids vif $(\mathrm{g}) / \mathrm{kg}$ MS & 150 & 163 & $13(8,7)$ \\
Gain de carcasse $(\mathrm{g}) / \mathrm{kg}$ MS & 95 & 99 & $4(4,2)$ \\
\hline
\end{tabular}

Tableau 7. Influence de la finesse de hachage et de l'éclatement des grains de l'ensilage de mais (39,6\% MS, $48 \%$ de grains) sur la quantité ingérée et les performances des taurillons (d'après Raymond et al 1985).

\begin{tabular}{|l|c|c|c|c|}
\hline Hachage & \multicolumn{2}{|c|}{ normal (6 mm) } & \multicolumn{2}{c|}{ insuffisant (13 mm) } \\
Eclateur & avec & sans & avec & sans \\
\hline Proportion de brins < 1 cm (\%) & 84 & 79 & 79 & 66 \\
Grains intacts en \% des grains totaux & 1,4 & 21,9 & 1,6 & 22,1 \\
Quantité ingérée totale (kg MS) & & & & \\
ensilage + 1 kg concentré & 7,06 & 7,04 & 6,94 & 6,97 \\
Gain de poids vif $(\mathrm{g} / \mathrm{j})$ & 1179 & 1197 & 1204 & 1128 \\
kg MS/kg de gain de poids vif & 5,99 & 5,88 & 5,76 & 6,18 \\
Grains entiers en \% de la MS des fèces & 1,9 & 8,1 & 1,8 & 6,8 \\
\hline
\end{tabular}

Tableau 8. Influence de la finesse de hachage du maïs sur la quantité volontairement ingérée par les vaches laitières (d'après De Brabender et al 1990).

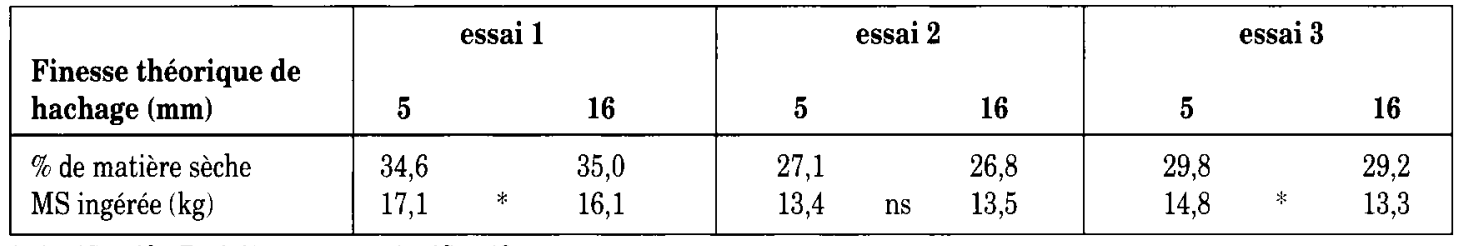

* significatif à $\mathrm{P} \leq 0,05 ;$ ns : non significatif

d'ensilage de maïs ingérées par les vaches laitières peuvent varier de plus de $10 \%$ d'un hybride à l'autre (J. Andrieu, communication personnelle). Il semble donc qu'il existe une variation d'ingestibilité liée à l'hybride, qu'il reste à préciser et surtout à essayer de prévoir.

Lorsque la plante de maïs est récoltée entre 25 et $40 \%$ de matière sèche et que les conditions de réussite de l'ensilage sont respectées (hachage fin, remplissage rapide, tassement correct, absence de terre, fermeture correcte du silo...), la qualité de conservation est déjà excellente et ne sera donc que très peu ou pas améliorée par l'addition de conservateurs qu'ils soient acides ou biologiques. L'emploi de ces conservateurs n'a donc pas, le plus souvent, modifié les performances animales (Baxter et al 1980, Buchanan-Smith et Tao 1981, Ely et al 1981, Moon et al 1981, El Hag et al 1982, Wittenberg et al 1983, Caar et al 1984). En revanche l'addition de conservateurs biologiques (ferments lactiques) améliore parfois la stabilité de l'ensilage en présence d'oxy- gène, donc après l'ouverture des silos (cf revue de Seal 1986), et peut donc être intéressante pour les ensilages distribués en été. Cette amélioration reste cependant inférieure à celle obtenue par l'addition de produits plus spécifiques et homologués pour cette utilisation tels Néosyl maïs.

Enfin, on sait que la teneur en matières azotées de l'ensilage de maïs est le plus souvent insuffisante pour assurer la couverture des besoins azotés non seulement des animaux, même de ceux maintenus au voisinage de l'entretien, mais aussi des microorganismes de leur rumen. L'ensilage de maïs doit donc être correctement complémenté en azote (et aussi en minéraux) pour atteindre son ingestibilité et sa valeur nutritive potentielles. La distribution des compléments énergétiques nécessaires pour couvrir les besoins des animaux à haut niveau de production va en revanche non seulement diminuer la quantité de matière sèche d'ensilage de maïs ingérée par suite des phénomènes de substitution bien connus mais peut 
Tableau 9. Ecart de digestibilité de la matière organique (mesurée - attendue) des rations d'ensilage de maïs complémenté avec 3 types d'aliment concentré, la valeur attendue étant calculée comme la moyenne pondérée des digestibilités des aliments composant la ration. Ecart correspondant entre la valeur énergétique effective (calculée à partir des performances des vaches laitières) et la valeur théorique (d'après Vérité et Dulphy 1981).

\begin{tabular}{|lcc|}
\hline Nature du concentré ${ }^{11)}$ & $\Delta$ dMO (en points) & $\Delta$ UFL/kg MS \\
\hline "Céréales" (38\% orge + 38\% mais) & $-3,5$ & $-0,04$ \\
"Pulpes" (81 \% pulpe de betterave) & $-1,1$ & $-0,01$ \\
"Son" (86\% son de blé) & 0 & 0 \\
\hline
\end{tabular}

"' L'aliment concentré représentait environ $20 \%$ de la MS ingérée.

aussi diminuer la digestibilité et la valeur nutritive de l'ensilage par suite des interactions digestives fourrages/concentrés. C'est ce qui ressort très bien des essais réalisés par Vérité et Dulphy (1981) qui ont comparé 3 types de concentrés pour complémenter des vaches laitières recevant comme ration de base un ensilage de maïs (tableau 9). Leurs résultats ont été obtenus avec des vaches produisant peu de lait (de l'ordre de $15 \mathrm{~kg}$ ) et recevant une ration contenant peu de concentrés (environ $20 \%$ sur la base de la matière sèche). Des diminutions de digestibilité beaucoup plus importantes ont été observées par Tyrell et Moe (1972), diminution de 3,0 et 3,5 points pour chaque augmentation de 1 du niveau d'alimentation (NA $=1$ pour l'entretien) respectivement avec des rations contenant 30 et $60 \%$ de concentrés. Il en résulte que les différences de digestibilité de l'énergie qui étaient de 2,4 points chez les vaches alimentées au niveau de l'entretien entre les régimes à 30 et $60 \%$ de concentré ne sont plus que de 1 point à 4 fois le niveau d'entretien (vaches produisant $35 \mathrm{~kg}$ de lait). Les différences de digestibilité de rations entraînées par celle des ensilages de maïs distribués, diminueront de même avec le niveau d'alimentation des vaches donc avec leur niveau de production.

Ce texte est une mise à jour de l'article "Variation factors in the nutritive value of silage maize" paru dans "Quality of silage maize, digestibility and zootechnical performance", International Seminar, Gembloux (Belgique), 29th nov. 1988, p 85-104.

\section{Références bibliographiques}

Andrieu J., 1969. Evolution entre la floraison et la maturité de la composition morphologique, chimique et de la production de matière sèche par hectare. Compte-rendu des Journées ITEB sur le maîs fourrage, 21-26.

Andrieu J., 1984. Prévision de la digestibilité et de la valeur énergétique des ensilages de maïs à partir de la composition de la plante à la mise en silo. Bull. Techn. CRZV Theix, INRA, 56, 5-8.

Andrieu J., Demarquilly C., 1974. Valeur alimentaire du maïs fourrage. II - Influence du stade de végétation, de la variété, du peuplement, de l'enrichissement en épis et de l'addition d'urée sur la digestibilité et l'ingestibilité des ensilages de maïs. Ann. Zootech., 23, 1-25.

Andrieu J., Demarquilly C., Dardenne P., Barrière Y., Lila M., Maupetit P., Riviere F., Femenias N., 1993. Composition and nutritive value of whole maize plants fed fresh to sheep. I. Factors of variation. Ann. Zootech., 42, 221-249.

Barrière Y., Gallois A., Huguet L., Traineau R. Malterre C., Bertin G., Micol D., Hoden A., Journet M., Mourguet A., 1985. Le maïs brown midrib plante entière. I - Caractéristiques générales. II Utilisation sous forme d'ensilage par les taurillons. III - Utilisation sous forme d'ensilage par les vaches laitières. Bull. Tech. CRZV Theix, INRA, 60, 43-58.

Baxter H.D., Montgomery M.J., Owen J.R., 1980. Formic acid treatment of wheat and corn silages. J. Dairy Sci., 63, 1291-1298.

Buchanan-Smith J.G., Tao Y.T., 1981. Effect of additives containing lactic acid bacteria and/or hydrolytic enzymes with an antioxidant upon the preservation of corn or alfalfa silage. Can. J. Anim. Sci., 61, 669-680.

Caar S.B., Hammes J.R., Moe A.J., Mc Gilliard M.L., 1984. Corn silage preservation with anhydrous ammoniac, live culture microbial or organic acidbased additives. J. Dairy Sci., 67, 1474-1481.

Coppock C.E., Stone J.B., 1968. Corn silage in the ration of dairy cattle. A review. Cornell Misc. Bull. 89.

Dardenne P., Andrieu J., Barrière Y., Biston R., Demarquilly C., Femenias N., Lila M., Maupetit R., Riviére F., Ronsin T.H., 1993. Composition and nutritive value of whole maize plants feds fresh to sheep. II. Prediction of the in vivo organic matter digestibility. Ann. Zootech., 42, 251-270.
La dMO et la valeur énergétique du mä̈s ensilage dépendent de la quantité et de la nature des concentrés distribués en complément. 
Davis W.E.P., Bowden D.M., 1969. Effect of growth stage at harvest on the nutritive value of a grain corn grown for silage. Can J. Plant Sci., 49, 361-370.

De Brabander D.L., De Boever J.L., Buysse F.X., 1990. The quality and utilisation of maize silage for dairy cattle. Conference British maize growers association, Maidenhead, U.K., 8 March 1990.

Deinum B., Steg A., Hof G., 1984. Measurement and prediction of digestibility of forage maize in the Netherlands. Anim. Feed Sci. Technol., 10, 301-313.

Demarquilly C., 1969. Valeur alimentaire du mais fourrage. I - Composition chimique et digestibilité du maïs sur pied. Ann. Zootech., 19, 45-51.

Dulphy J.P., Michalet-Doreau B., 1981. Prévision de lingestibilité des ensilages d'herbe. in "Prévision de la valeur nutritive des aliments des ruminants". Ed. INRA Publications, Route de St-Cyr, 78000 Versailles, p. 169-187.

El Hag H.G., Vetter R.L., Kenealy M.D., 1982. Effects of silage addition on fermentation characteristics of corn silage and performance of feedlot heifers. J. Dairy Sci., 65, 259-266.

Ely L.O., Moon N.J., Sudweeks E.M., 1981. Inoculation with Lactobacillul plantarum of alfalfa, corn, sorghum and wheat silage. J. Dairy Sci., 64, $2378-2387$

Fisher C.J., Fairey N.A., 1982. The effect of planting density on the nutritive value of corn silage for lactating cows. Can. J. Anim. Sci., 62, 1143-1148.

Gabon G., 1987. Ensilage de maïs : la coupe fine rapporte plus qu'elle ne coûte. Perspectives agricoles. Supplément au n¹13, 53-57.

Gordon C.H., Derbyshire J.C., Van Soest P.J., 1968. Normal and late harvesting of corn for silage. J. Dairy Sci., 51, 1258-1263.

Gross F., 1980. Content and in vivo digestibility (sheep) of nutrients in maize varieties harvested at different stages for silage. in "Improvement of quality traits of maize for grain and silage use. Ed. Pallmer W.G. and Phipps R.H., p. 429-445.

Harris C.E., 1965. The digestibility of fodder maize and maize silage. Expl. Agric., 1, 121-123.

Hillman D., 1969. Supplementing corn silage. J. Dairy Sci., 52, 859-870.

Huber J.T., Graf G.C., Engel R.W., 1965. Effect of maturity on nutritive value of corn silage for lactating cows. J. Dairy Sci., 48, 1121-1123.

Huber J.T., Sandy R.A., Miller C.N., Polan C.E., 1966. Recut corn silage for dairy cattle. J. Anim. Sci., 25, 914.

Johnson R.R., Mcclure K.E., 1968. Corn plant maturity. IV -Effects on digestibility of corn silage in sheep. J. Anim. Sci., 27, 535-540.
Malterre C., 1976. Utilisation du maïs sous différentes formes pour la production de viande de jeunes bovins. Bull. Techn. CRZV Theix, INRA, 24, 63-74

Miller C.N., Polan C.E., Sandy R.A., Huber J.J., 1969. Effect of altering the physical form of corn silage on utilization by dairy cattle. J. Dairy Sci., 52 , 1955-1960.

Moon N.J., Ely L.O., Sudweeks E.M., 1981. Fermentation of wheat, corn, alfalfa silages inoculated with Lactobacillus acidophilus and candida sp. at ensiling. J. Dairy Sci., 64, 807-813.

Noller C.H., Burns J.C., Hill D.L., Rhykerd C.L., Rumsey T.S., 1963. Chemical composition of green and preserved forage and the nutritional implications. Proc. 9th Intern. Grassland Congr. Sao Paulo, 611-614.

Owen F.G., 1967. Factors affecting nutritive value of corn and sorghum silage. J. Dairy Sci., $50,404-416$

Raymond F., 1985. Les variations de consommation et de performances induites par la plante entière de maïs. Colloque maïs-ensilage. Rennes 29 et 30 Mai 1985. Tome II.

Raymond F., Citron G., Sagot L., 1986. Effet de la qualité de l'ensilage de mais sur les performances des taurillons. Perspectives Agricoles. Supplément au ${ }^{\circ} 105,33-43$.

Rémond B., Journet M., 1977. Utilisation de deux ensilages de maïs de composition morphologique différente par les vaches laitières. Bull. Tech. CRZV Theix, INRA, 29, 49-52.

Rivière F., 1990. Valeur nutritive et valorisation de l'ensilage de maïs par les vaches laitières. In : «Maïs ensilage et céréales pour les ruminants. ITCF et AGPM. Angers, 4 Avril 1990, p44-56.

Seale D.R., 1986. Bacterial inoculants as silage additives. J. Applied Bacteriol. Symposium, Suppl., 9S- $26 \mathrm{~S}$.

Tyrell H.F., Moe P.W., 1972. Net energy value for lactation of a high and low concentrate ration containing corn silage. J. Dairy Sci., 55, 1106-1112.

Vérité R., Dulphy J.P., 1981. Effet de la nature de l'aliment concentré sur l'ingestion et les performances des vaches laitières. Bull. Techn. CRZV Theix, INRA, 45, 15-21.

Wilkinson J.M., Phipps R.H., 1979. The developpment of plant components and their effects on the composition of fresh and ensiled forage maize. 2 - The effect of genotype, plant density and date of harvest on the composition of maize silage. J. Agric. Sci., 92, 485-491.

Wittenberg K.M., Ingalls J.R., Devlin T.J., 1983. The effect of lactobacteria inoculation on corn silage preservation and feeding value for growing beef animals and lambs. Can. J. Anim. Sci., 63, 917-924. 


\section{Summary}

Factors governing the nutritional value of silage maize

The morphological composition of the maize plant evolves up to the glazed grain stage (35\% dry matter (DM) in the whole plant), when the grain reaches its physiological maturity, making up 40 to $50 \%$ of the DM. The chemical composition changes little after flowering except for starch content, which rises at the expense of water soluble carbohydrates. The digestibility of the organic matter (dMO) and the ingestibility of a particular maize measured in sheep hardly changed after the milky stage. These averaged respectively $71.5 \pm 2.2 \%$ and $52.5 \pm 7.2 \mathrm{~g} \mathrm{DM} / \mathrm{kg} P 0.75$ for the 20 maize plants we studied between 1964 and 1971. The dMO averaged 72.6 \%and ranged from 67 to $78 \%$ for the 15 normal hybrids studied by the "digestibility club" in 1987 and 1988. Apart from the bm maizes, the variations in digestiblity observed among varieties grown the same year in any particular location were slight and of the same order of magnitude as those observed for the same variety grown in different locations and in different years.

Owing to its high DM, its non-limiting soluble carbohydrate content, and above all its low buffering capacity, maize keeps very well as silage if finely chopped. The dOM of silage was the same as that of the fresh cut plant. Its ingestibility in sheep was also closely similar, provided the silage was properly supplemented, particularly for nitrogen. However, dry matter intake by cattle increased with DM content up to approximately $35 \%$.

The DM content of maize silage is thus the essential factor governing net energy intake in cattle. In fattening cattle, though it has been shown that live weight gain increases with DM content in sila. ge maize, feeding efficiency and so probably energy value remains constant. This seems to be true also for dairy cattle.

For the same DM content, intake and performance of fattening and dairy cattle, and the feeding efficiency and therefore the energy value varied in the same way as the digestibility of the silage.

Animal performance was scarcely affected by devices such as mesh screens or rollers that reduce the amounts of whole grains in faeces. However, the effective energy value of the silage maize based feed, calculated from performance, depen. ded on the nature and the amount of energy supplement intake.

DEMARQUILLY C., 1994. Facteurs de variation de la valeur nutritive du mais ensilage. INRA Prod. Anim., 7 (3), 177-189. 